\title{
Generating BDDs for Symbolic Model Checking in CCS
}

\author{
Reinhard Enders, Thomas Filkorn, Dirk Taubner \\ Siemens AG, Corporate Research and Development (ZFE IS INF2) \\ Otto-Hahn-Ring 6, W-8000 München 83, F.R. Germany \\ email: \{reinhard, thomas\%apollo21, taubner \} Qztivax.uucp
}

\begin{abstract}
Finite transition systems can easily be represented by binary decision diagrams (BDDs) through the characteristic function of the transition relation. Burch et al. have shown how model checking of a powerful version of the $\mu$-calculus can be performed on such BDDs.

In this paper we show how a BDD can be generated from elementary finite transition systems given as BDDs by applying the CCS operations of parallel composition, restriction, and relabelling. The resulting BDDs only grow linearly in the number of parallel components. This way bisimilarity checking can be performed for processes out of the reach of conventional process algebra tools.
\end{abstract}

\section{Introduction}

A binary decision diagram as described by Bryant [1] is a normal form representation of boolean functions $f: \mathbb{B}^{n} \rightarrow \mathbb{B}$, where $\mathbb{B}=\{0,1\}$. It is often much smaller than other normal form representations. Moreover boolean operations can be applied efficiently.

Transition systems have states and transitions leading from one state via some action to another state. They underly many semantics for concurrent systems, e.g. bisimulation equivalence for CCS [10]. They also underly semantics of modal logics, which allow to reason about concurrent systems.

Burch, Clarke, McMillan, Dill, and Hwang [2] have shown how model checking of a powerful version of the $\mu$-calculus including existential quantification and $\lambda$-abstraction can be performed for a boolean domain using BDDs for internal representation. They call the approach symbolic model checking. They also indicate how other finite domains can be treated through binary encoding.

In particular for a finite transition system the set of states $S$ and the set Act of actions can be encoded as subsets of $\mathbb{B}^{\left.\log _{2}|S|\right\rceil}$ and $\mathbb{B}^{\log _{2}|A c t| \eta}$ respectively. The transition relation $D \subseteq S \times A c t \times S$ can be encoded as a function $\chi: \mathbb{B}^{\left.2 \cdot\left|\log _{2}\right| S \mid\right\rceil+\left\lceil\log _{2}|A c t| \mid\right.} \rightarrow \mathbb{B}$ which in turn can be represented as a BDD. Burch et al. show how weak and strong bisimilarity can be expressed as formulas in their $\mu$-calculus and hence can be checked for transition systems given as BDDs.

However they do not indicate how the BDDs are generated. If they were generated from the list of state-action-state triples of the transition relation (which is straightforward) the approach would immediately suffer the well-known explosion problem, namely that a system of $N$ parallel components, with $n$ states each, may have $n^{N}$ transitions.

The question arises whether the BDD which represents the transition system of the compound system could be generated more efficiently directly from the $N$ components given as BDDs without enumerating all resulting transitions. This question is the topic of this paper. It is answered in the following way: We give a certain encoding of transition systems as BDDs. We show how the CCS [10] operators of parallel composition, restriction, and relabelling can be applied to BDDs. We prove that for a fixed set of actions the BDD for representing $N$ parallel communicating processes grows only linearly in $N$. Worst case boundaries for the size of BDDs are rare in literature, hence this bound is interesting. However it has to be pointed out that it concerns only the resulting BDD which represents the system of $N$ parallel component processes. It does not concern intermediate BDDs which are used during the generation or during the model checking. Nevertheless a benchmark example shows that bisimilarity checking can be performed for systems out of the reach of conventional tools such as $[3,4$, $7,9,6]$. 
Note that the $\mu$-calculus of [2] is more powerful than that of e.g. [5, 11]. Hence all formulas checked with the latter approaches can be checked in the framework of this paper. We expect to be able to handle larger transition systems.

On the other hand it should be noted that BDDs are efficient only in a heuristic sense, the worst case may still be catastrophic. For circuit verification experience has shown that indeed BDDs can serve as an efficient representation in many practical cases. This paper gives evidence that this is also the case for verification of parallel processes. Possible applications are communication protocols, operating system tasks, and distributed control systems.

Before we start with the technical part of this paper let us explain with an example the basic idea, i.e. how to represent relations by BDDs and why this is promising for the parallel composition of transition systems. Consider the relation $D$ of Fig. 1 where $D \subseteq S \times S$ for $S=\{0,1,2,3\}$. We omit the actions for the moment. An obvious boolean encoding of $S$ is $0 \mapsto 00,1 \mapsto 01,2 \mapsto 10,3 \mapsto 11$. The relation $D$ may then be represented by a function $\chi: \mathbb{B}^{4} \rightarrow \mathbb{B}$, such that $\chi\left(r_{1}, r_{2}, s_{1}, s_{2}\right)=1$ if and only if the state encoded by $r_{1} r_{2}$ has an edge to the state encoded by $s_{1} s_{2}$. This boolean function $\chi$ can in turn be represented as a BDD.

$D$

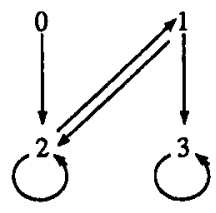

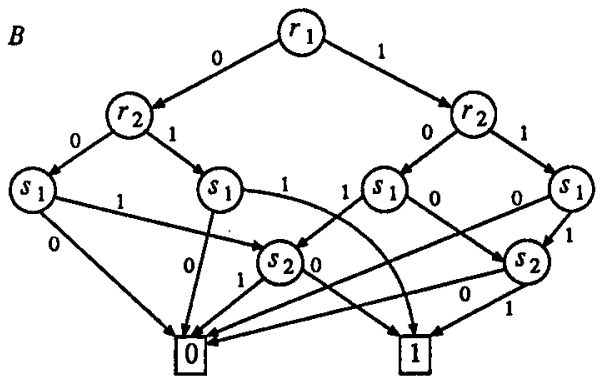

Figure 1: A relation (left) and its BDD representation (right)

For a given set of boolean variables which are totally ordered by $\prec$ a BDD is a rooted directed acyclic graph, each node is either terminal, then it is labelled by a truthvalue and has no successor, or it is nonterminal, then it is labelled by some variable and has two successors which are terminal or labelled by a larger $(\prec)$ boolean variable. One successor corresponds to 0 , the other to 1 .

Taking the ordering $r_{1} \prec r_{2} \prec s_{1} \prec s_{2}$ the function $\chi$ is represented by the BDD $B$ given in Fig. 1 . Now assume we want to represent the relation ${ }^{1}$

$$
\left\{\left\langle\left\langle r, r^{\prime}\right\rangle,\left\langle s, s^{\prime}\right\rangle\right\rangle \in S \times S \mid\langle r, s\rangle \in D \wedge\left\langle r^{\prime}, s^{\prime}\right\rangle \in D\right\}
$$

as a BDD $C$. We can take a copy $B^{\prime}$ of $B$ with fresh variables $r_{1}^{\prime}, r_{2}^{\prime}, s_{1}^{\prime}, s_{2}^{\prime}$ and calculate $C$ as $B \wedge B^{\prime}$ where the conjunction of BDDs is as described in [1].

However the size of $C$ depends on the chosen variable ordering. If $r_{1} \prec r_{2} \prec s_{1} \prec s_{2} \prec r_{1}^{\prime} \prec r_{2}^{\prime} \prec$ $s_{1}^{\prime} \prec s_{2}^{\prime}$ is chosen it has 20 nodes, approximately $2 \cdot|B|$, where $|B|$ denotes the number of nodes in $B$. It is formed simply by attaching $B^{\prime}$ to the 1-exit of $B$. If, on the other hand, $r_{1} \prec r_{2} \prec r_{1}^{\prime} \prec r_{2}^{\prime} \prec s_{1} \prec$ $s_{2} \prec s_{1}^{\prime} \prec s_{2}^{\prime}$ is chosen it has 47 nodes (approximately $|B|^{2} / 2$ ).

This ends our introductory example. We wanted to indicate that if the ordering of variables is chosen carefully the BDD only grows additively while the number of elements in the represented relation grows multiplicatively. However be aware that the encoding as presented in Section 4 uses a different ordering in order to also serve the asynchronous case well.

\footnotetext{
${ }^{1}$ This relation resembles the synchronization part of parallel composition, cf. the next section. In general, of course, one wants to combine two relations and not twice the same.
} 


\section{Operators on transition systems}

Our language for composing parallel processes is taken from the process algebra CCS [10]. Let Act be the finite set of actions which contains the invisible action $\tau$ and all other actions in two copies, $a$ and $\bar{a}$, which are complementary, i.e. $\overline{\bar{a}}=a$. A transition system $T=\langle S, D, z\rangle$ has states $S$, initial state $z \in S$, and transitions $D \subseteq S \times$ Act $\times S$ leading from one state with some action to another state. Throughout we assume $S$ to be finite.

Next we give operators on transition systems which correspond to CCS parallel composition, restriction, and relabelling. In [12] it is shown that they are correct (consistent) in the following sense. Given two closed CCS terms $P_{1}$ and $P_{2}$ and let $T_{1}, T_{2}$ be their respective transition systems according to the transitional semantics [10], then $T_{1} \mid T_{2}$ as defined below is strongly bisimilar to the transition system of $P_{1} \mid P_{2}$ according to the transitional semantics. Similar results hold for restriction and relabelling.

The CCS parallel composition for given $T_{1}=\left\langle S_{1}, D_{1}, z_{1}\right\rangle$ and $T_{2}=\left\langle S_{2}, D_{2}, z_{2}\right\rangle$ is defined as $T_{1} \mid T_{2}=\left\langle S_{1} \times S_{2}, D,\left\langle z_{1}, z_{2}\right\rangle\right\rangle$ where

$$
\begin{aligned}
& D=\left\{\left\langle\left\langle r_{1}, r_{2}\right\rangle, \alpha,\left\langle s_{1}, s_{2}\right\rangle\right\rangle \mid\right.\left\langle r_{1}, \alpha, s_{1}\right\rangle \in D_{1} \wedge r_{2}=s_{2} \vee\left\langle r_{2}, \alpha, s_{2}\right\rangle \in D_{2} \wedge r_{1}=s_{1} \\
&\left.\vee \quad \alpha=\tau \wedge \exists a \neq \tau:\left\langle r_{1}, a, s_{1}\right\rangle \in D_{1} \wedge\left\langle r_{2}, \bar{a}, s_{2}\right\rangle \in D_{2}\right\} .
\end{aligned}
$$

The condition $\left\langle r_{1}, \alpha, s_{1}\right\rangle \in D_{1} \wedge r_{2}=s_{2}$ and its symmetric version represent an asynchronous move, only one component proceeds. On the other hand the condition $\left\langle r_{1}, a, s_{1}\right\rangle \in D_{1} \wedge\left\langle r_{2}, \bar{a}, s_{2}\right\rangle \in D_{2}$ represents a synchronous move. The latter in a simplified version has already been discussed in the introduction.

Let $T=\langle S, D, z\rangle$ be given. Restriction of a subset $A \subseteq A c t-\{\tau\}$ is defined as

$$
T \backslash A=\langle S, \quad\{\langle r, \alpha, s\rangle \in D \mid \alpha \notin A \wedge \bar{\alpha} \notin A\}, \quad z\rangle .
$$

Relabelling of a visible action $a(a \neq \tau)$ into the action $b$ is defined as $T[b / a]=\left\langle S, D^{\prime}, z\right\rangle$ where

$$
D^{\prime}=\{\langle r, \beta, s\rangle \mid \exists \alpha:\langle r, \alpha, s\rangle \in D \wedge(\beta=\alpha \notin\{a, \bar{a}\} \vee \beta=b \wedge \alpha=a \vee \beta=\bar{b} \wedge \alpha=\bar{a})\} .
$$

\section{Symbolic model checking using BDDs}

Burch, Clarke, McMillan, Dill, and Hwang [2] show how model checking can be performed using BDDs. They call their approach 'symbolic' as it proceeds without supplying an interpretation for the individual variables. The result of model checking a formula is a BDD which represents the result of the formula for all interpretations. Given a particular interpretation taking choices according to the values of the variables under this interpretation leads from the root of the BDD to a truthvalue. This is the truthvalue of the formula under the particular individual variable interpretation. Additionally in [2] BDDs are used to represent relations internally with the intention that this is more efficient than the corresponding lists of tupels.

The version of the $\mu$-calculus used in [2] includes individual variables, $n$-ary relational variables $(n>0)$, existential quantification, abstraction ( $\lambda$-binding) of individual variables, and fixpoint binding of relational variables. The reader is referred to [2] for the details of syntax and semantics.

In general, given a structure of a domain and interpretations $I_{P}$ and $I_{D}$ of relational and individual variables, model checking performs the task of checking, whether a formula $F$ is true in this structure (i.e. $\mathcal{D}(F)\left(I_{P}\right)\left(I_{D}\right)=1$ ), in other words, whether the structure is a model for $F$.

From a different point of view the model checker of Burch et al. may be seen as a certain approach to work with relations which are represented as BDDs. The actual model checking of a formula $F$ proceeds by first calculating as a BDD the relation which contains a tuple $\left\langle d_{1}, \ldots, d_{n}\right\rangle$ iff $F$ is true for the individual variable interpretation which maps the first variable to $d_{1} \ldots$ and the last variable to $d_{n}$. The model checking is finished (and yields a truthvalue) by instantiating this BDD to a particular individual variable interpretation.

However the interpretation of the relational variables have to be supplied before the symbolic model checker can start. They have to be supplied as BDDs. In particular for model checking CCS-terms 
[2, Sect. 8] the transition relations of the transition systems to be checked have to be supplied by the relational variable interpretation, i.e. as BDDs. Burch et al. leave open where the BDDs for the transition relations come from. The following two sections propose an approach for generating these BDDs.

\section{Encoding of transition systems as BDDs}

In principle given a certain enumeration $e_{0}, e_{1}, \ldots, e_{|S|-1}$ of a finite set $S$ the boolean encoding is obvious, one needs $\# S:=\left\lceil\log _{2}|S|\right\rceil$ boolean variables and lets $s_{1}, \ldots, s_{\# S}$ denote $e_{i}$ where $i$ is the number one gets when interpreting $s_{1} \ldots s_{\# S}$ as a binary digit.

We will use this encoding for the state sets of elementary ${ }^{2}$ transition systems and for the set of actions. For the latter we additionally assume that $A c t$ is enumerated $\tau, a, b, \ldots, \bar{a}, \bar{b}, \ldots$, i.e. that $\tau$ is encoded as all zeros and any visible action $a$ as $0 x_{2} \ldots x_{\# A}$ and its complement $\bar{a}$ as $1 y_{2} \ldots y_{\# A}$ such

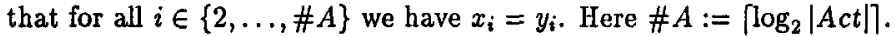

In order to work with BDDs one has to fix a global ordering on the boolean variables used to encode the information. This ordering has great influence on the size of the BDDs. If nothing is known on the structure of a relation to be represented as a BDD not much can be done. However in our case we can use knowledge about the transition relation and in particular about the operations performed on them, most importantly the parallel composition.

For explanation let us consider a binary relation $D \subseteq S \times S$ as in the introduction. If we need \#S bits to encode $S$ we need $2 \cdot \# S$ bits to encode $D$, say $r_{1}, \ldots, r_{\# S}$ to encode the first element of a pair in $D$ and $s_{1}, \ldots, s_{\# S}$ for the second. In the introduction we demonstrated for the synchronization case of parallel composition of two such relations that we get small BDDs if all variables of one relation are ordered before those of the other.

However in the asynchronous case where only one component proceeds while the other (say the first) stays in its state we have to check for $\left\langle\left\langle r, r^{\prime}\right\rangle,\left\langle s, s^{\prime}\right\rangle\right\rangle$ whether $r=s \wedge\left\langle r^{\prime}, s^{\prime}\right\rangle \in D$, i.e. we have to check whether $r_{1}=s_{1} \wedge r_{2}=s_{2} \wedge \ldots \wedge r_{\# S}=s_{\# S}$. But with the variable ordering $r_{1} \prec r_{2} \prec \ldots r_{\# S} \prec$ $s_{1} \prec \ldots \prec s_{\# S}$ for the bits of a relation the BDD for this check explodes.
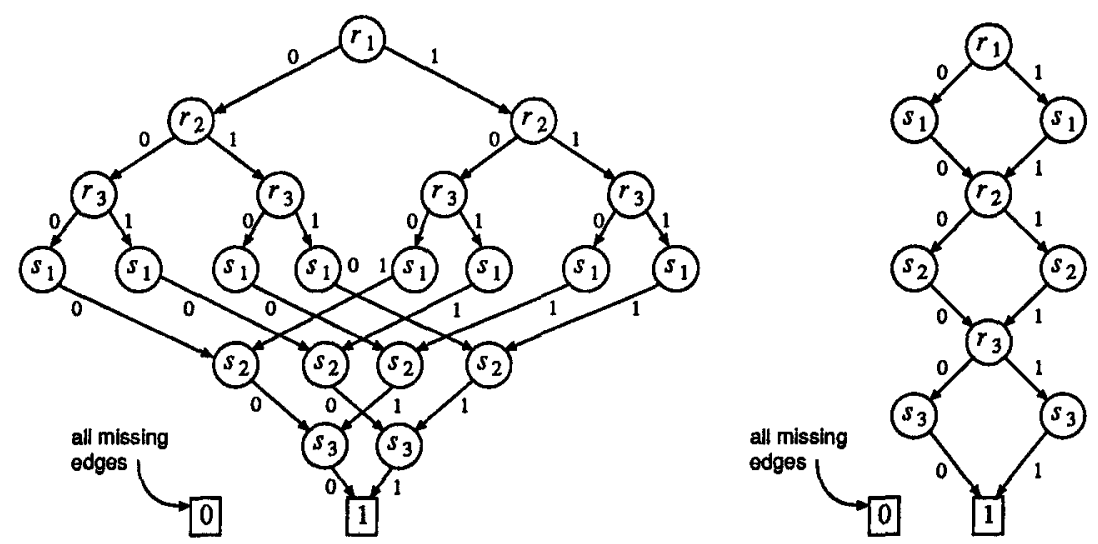

Figure 2: BDD for $\left(r_{1}=s_{1}\right) \wedge\left(r_{2}=s_{2}\right) \wedge\left(r_{3}=s_{3}\right)$ with poor (left) and good (right) variable ordering

Figure 2 gives an example for $\# S=3$. In general it has $\Omega\left(2^{\# S}\right)$ nodes. Therefore we choose a different ordering, namely

$$
r_{1} \prec s_{1} \prec r_{2} \prec s_{2} \prec \ldots \prec r_{\# S} \prec s_{\# S} .
$$

\footnotetext{
${ }^{2}$ We call a transition system elementary if it is not formed by parallel composition, restriction, or relabelling but is given as a list of transitions.
} 
With this improved ordering the BDD for the above example is as given on the right of Figure 2, i.e. it grows only linearly.

We are now ready to present our encoding for a transition relation $D \subseteq S \times A c t \times S$. We choose the variable ordering

$$
a_{1} \prec a_{2} \prec \ldots a_{\# A} \prec r_{1} \prec s_{1} \prec r_{2} \prec s_{2} \prec \ldots \prec r_{\# S} \prec s_{\# S}
$$

where $a_{1} \ldots a_{\# A}$ encodes the action of the transition, $r_{1} \ldots r_{\# S}$ encodes the source state of the transition, and $s_{1} \ldots s_{\# S}$ encodes the target state. We always understand that $00 \ldots 0$ is the initial state. Fig. 3 gives an example transition system and Fig. 4 shows the representation of its transition relation as a BDD.

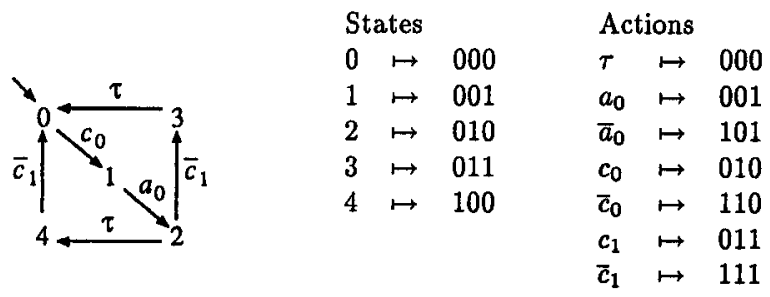

Figure 3: A transition system and the encoding of its states and actions

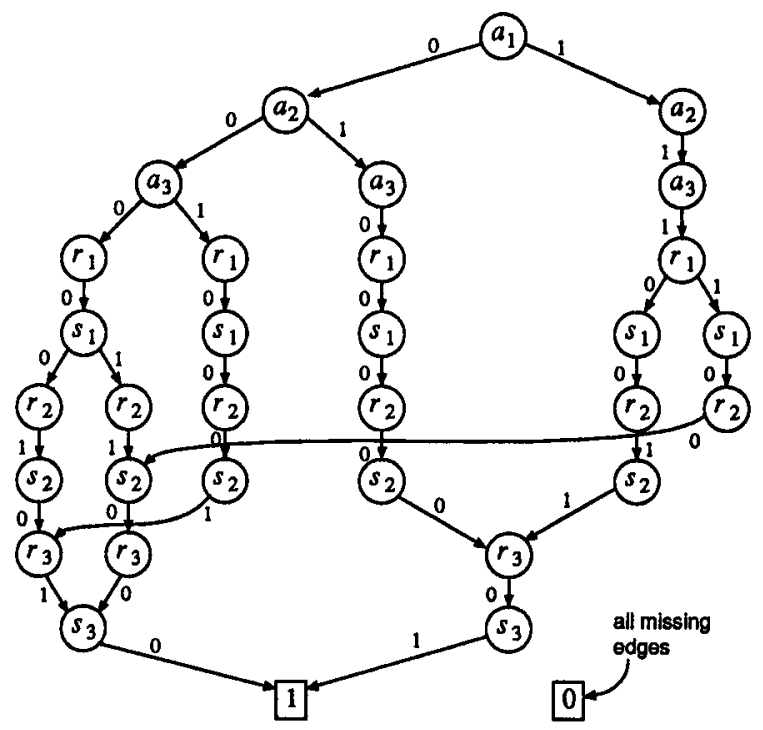

Figure 4: BDD encoding of the transition relation of the system of Fig. 3

The reason to put the bits for the actions above those for the states is that in the worst case this leads to only $|A c t| r_{1}$-labelled nodes in the BDD, each corresponding to one action. If the bits were below those of the states the worst case of $a_{1}$-labelled nodes would be $2^{|A c t|}$, each corresponding to a subset of Act. However this is an intuitive reason only. As it stands it does not imply that the resulting BDD is smaller. 


\section{Operators on BDDs}

In this section we describe the operators of CCS parallel composition, restriction, and relabelling on BDDs. Note that this comprises the practically most important case of a parallel composition of $N$ processes

$$
\left(P_{1}\left|P_{2}\right| \ldots \mid P_{N}\right) \backslash A
$$

where the restriction imposes the wanted synchronization.

We assume that the elementary processes (transition systems) are given as BDDs. The BDD for an elementary transition system may easily be formed from the list of triples in the transition relation as follows. Each transition $\langle r, a, s\rangle$ which has encodings $b r_{1}, \ldots, b r_{\# S}, b a_{1}, \ldots, b a_{\# A}$, and $b s_{1}, \ldots, b s_{\# S}$, is considered as one BDD with only the path $a_{1} \stackrel{b a_{2}}{\rightarrow} a_{2} \stackrel{b a_{2}}{\rightarrow} \ldots a_{\# A} \stackrel{b a_{\# A}}{\rightarrow} r_{1} \stackrel{b r_{1}}{\rightarrow} s_{1} \stackrel{b s_{1}}{\rightarrow} \ldots r_{\# S} \stackrel{b r_{\# S}}{\rightarrow} s_{\# S} \stackrel{b s_{\# S}}{\rightarrow} 1$ leading to 1. All other branches directly lead to 0 . The BDD for the transition relation is calculated by performing the disjunction (cf. [1]) of all these BDDs. In the worst case this yields a BDD with approximately $2^{2 \cdot \# S+\# A}$ nodes.

\section{CCS parallel composition}

Let $B_{1}$ and $B_{2}$ be two BDDs representing transition relations $D_{1}$ and $D_{2}$ over the same set of actions but over disjoint sets of states encoded as described in the previous section. The BDD $B_{1} \mid B_{2}$ representing the transition relation of the parallel composition is calculated as follows.

Let $a_{1}, \ldots, a_{\# A}$ be the boolean variables for the actions in both, $B_{1}$ and $B_{2}$. Let $r_{i}, s_{i}(i \in$ $\left.\left\{1, \ldots, \# S_{1}\right\}\right)$ and $r_{j}^{\prime}, s_{j}^{\prime}\left(j \in\left\{1, \ldots, \# S_{2}\right\}\right)$ be the boolean variables for the source and target states of $D_{1}$ and $D_{2}$ respectively. According to the previous section we have the following ordering dependencies

and

$$
\begin{aligned}
& a_{1} \prec \ldots \prec a_{\# A} \prec r_{1} \prec s_{1} \prec \ldots \prec r_{\# S_{1}} \prec s_{\# S_{1}} \\
& a_{1} \prec \ldots \prec a_{\# A} \prec r_{1}^{\prime} \prec s_{1}^{\prime} \prec \ldots \prec r_{\# S_{2}}^{\prime} \prec s_{\# S_{2}}^{\prime} .
\end{aligned}
$$

We additionally impose that

$$
s_{\# S_{1}} \prec r_{1}^{\prime}
$$

this leaves the previous orderings and hence $B_{1}, B_{2}$ unchanged.

Note that this choice of combined ordering ensures that the BDD for $B_{1} \mid B_{2}$ again fulfills our encoding convention for transition systems, i.e. we use the first \#A variables for the actions and than alternatingly one bit for the source and one for the target state of the transition.

We now may calculate $B_{1} \mid B_{2}$ as

$$
B_{1} \wedge S_{t a b_{2}} \vee B_{2} \wedge \operatorname{Stab}_{1} \vee C
$$

where the missing components are explained below. The $\Lambda$ and $\vee$ are operations on BDDs as described in [1]. Stab $b_{1}$ is the BDD for $r_{1}=s_{1} \wedge \ldots \wedge r_{\# S_{1}}=s_{\# S_{1}}$ (cf. Fig. 2). It corresponds to the condition that the first component stays in its state ( $S t a b_{2}$ is analogous). Let $E$ be the BDD calculated as

$$
\left.\left.\left.\left.B_{1}\right|_{a_{1}=0} \wedge B_{2}\right|_{a_{1}=1} \vee B_{1}\right|_{a_{1}=1} \wedge B_{2}\right|_{a_{1}=0} \text {. }
$$

$\left.B_{1}\right|_{a_{1}=0}$ denotes the subgraph of $B_{1}$ one gets when restricting the variable $a_{1}$ to 0 (see [1] for details) again $\wedge$ and $\vee$ are operations on BDDs. The BDD $E$ expresses the condition that complementary actions match. The last component $C$ of the parallel composition above is calculated as

$$
\left(a_{1}=0\right) \wedge \ldots \wedge\left(a_{\# A}=0\right) \wedge\left(\exists a_{2} \exists a_{3} \ldots \exists a_{\# A} E\right) .
$$

Here $\exists a_{i} G$ for some BDD $G$ is short for $\left.\left.G\right|_{a_{i}=0} \vee G\right|_{a_{1}=1}$, i.e. it represents an existential quantification of the boolean variable $a_{i}$. The existential quantification of $a_{2}, \ldots, a_{\# A}$ can be implemented directly as a BDD operator. Applied to $E$ it yields a BDD without nodes labelled by variables $a_{1}, \ldots, a_{\# A}$. The first part of $C$ puts this BDD below the encoding of $\tau$.

\section{Restriction}

Given a BDD $B$ representing the transition relation of $T$ and a BDD $C$ for the set of actions $A$ (it may be generated straightforward from the list of actions in $A$ ) the BDD for the transition relation of $T \backslash A$ is simply $B \wedge \neg\left(\left.\left.C\right|_{a_{1}=0} \vee C\right|_{a_{1}=1}\right)$, where $\wedge, \vee, \neg$, and $\left.\right|_{a_{1}=b}$ on BDDs are as described in [1]. 


\section{Relabelling}

For $B$ as above let the binary encoding of actions $a$ and $b$ be $b a_{1}, \ldots, b a_{\# A}$ and $b b_{1}, \ldots, b b_{\# A}$ respectively. The BDD for the transition relation of $T[b / a]$ is

$$
\begin{gathered}
B \wedge \neg\left(a_{2}=b a_{2} \wedge \ldots \wedge a_{\# A}=b a_{\# A}\right) \\
\left.\left.\vee\left(a_{2}=b b_{2} \wedge \ldots \wedge a_{\# A}=b b_{\# A}\right) \wedge B\right|_{a_{2}=b a_{2}}\right|_{a_{3}=b a_{3}}|\ldots|_{a_{\# A}=b a_{\# A}} .
\end{gathered}
$$

\section{Complexity of resulting BDDs}

We have the following results on the size of the generated BDDs.

Theorem 6.1 Let $T_{i}=\left\langle S_{i}, D_{i}, z_{i}\right\rangle$ for $i \in\{1, \ldots, N\}$ be transition systems where the transition relation is represented as $B D D B_{i}$ according to Section 4 .

The number of nodes of the BDD $B:=B_{1}\left|B_{2}\right| \ldots \mid B_{N}$ is

$$
O\left(2^{|A c t|} \cdot \sum_{i=1}^{N}\left|S_{i}\right|^{2}\right) \text {. }
$$

Proof We will use $\vec{a}, \vec{r}_{i}, \vec{s}_{i}$ as abbreviations such that $\vec{a}=\left\langle a_{1}, \ldots, a_{\# A}\right\rangle$ is the variable vector encoding the actions of $A c t$ and such that $\vec{r}_{i}=\left\langle r_{i, 1}, \ldots, r_{i, \# S_{i}}\right\rangle$ and $\vec{s}_{i}=\left\langle s_{i, 1}, \ldots, s_{i, \# S_{i}}\right\rangle$ are the variable vectors encoding source and target state of the transition relation $D_{i}$. The BDD $B_{i}$ ranges over the variables $\vec{a}, \vec{r}_{i}, \vec{s}_{i}$ and the BDD $B$ over the variables $\vec{a}, \vec{r}_{1}, \vec{s}_{1}, \ldots, \vec{r}_{N}, \vec{s}_{N}$. The variable ordering is $\vec{a} \prec \vec{r}_{1}, \vec{s}_{1} \prec \ldots \prec \vec{r}_{N}, \vec{s}_{N}$ where the ordering within $\vec{r}_{i}, \vec{s}_{i}$ is as described in Section 4.

For the proof we have a layered view of $B$. Let us say that all variables for states of transition system $T_{l}$ belong to level $l$.

For instances $\overrightarrow{b a} \in \mathbb{B}^{\# A}$ and $\vec{b} \vec{r}_{i}, \overrightarrow{b s_{i}} \in \mathbb{B}^{\# S_{i}}$ let $\sigma_{l}$ denote the instantiation $\left\langle\overrightarrow{b a}, \vec{b} \vec{r}_{1}, \overrightarrow{b s_{1}}, \ldots, \vec{b} \vec{r}_{l-1}\right.$, $\left.\overrightarrow{b s_{l-1}}\right\rangle$. Let $\sigma_{l}(B)$ denote the BDD one gets by instantiating $B$ to $\sigma_{l}$, i.e.

$$
\sigma_{l}(B):=\left.B\right|_{\vec{a}=b \vec{a}, \vec{r}_{1}=\vec{b} \vec{r}_{1}, \vec{s}_{1}=\vec{b} \vec{s}_{1}, \ldots, \vec{r}_{i-1}=b \vec{r}_{i-1}, \vec{s}_{l-1}=b \vec{s}_{i-1}} .
$$

This BDD $\sigma_{l}(B)$ denotes a boolean function of arity $2 \cdot \sum_{i=1}^{N} \# S_{i}$.

To count the nodes of $B$ on level $l$ we determine the number of different such functions one gets by varying $\sigma_{l}$ over all instances. This number is called width $(l)$. Since BDDs are a normal form for boolean functions and they are reduced (i.e. have no two isomorphic subgraphs) [1] this number immediately gives an upper bound for the number of nodes in $B$ which are labeled with variables of level $l$. For $j \in\left\{1, \ldots, \# S_{l}\right\}$ this bound is width $(l) \cdot 2^{2 \cdot(j-1)}$ for the label $r_{l, j}$ and width $(l) \cdot 2^{2 \cdot(j-1)+1}$ for the label $s_{l, j}$. Note that we do not make any assumptions about the part of $B$ within level $l$ hence we have to allow the exponential growth in this part. Knowing an upper bound $W$ for $\max l$ width $(l)$ we get an upper bound for $\{B \mid$, the number of nodes in $B$.

$$
\begin{aligned}
|B| & \leq 2^{\# A}+\sum_{l=1}^{N} \sum_{j=1}^{\# S_{l}} \text { width }(l) \cdot\left(2^{2 \cdot(j-1)}+2^{2 \cdot(j-1)+1}\right) \\
& \leq 2^{\# A}+\sum_{l=1}^{N} \text { width }(l) \cdot 2^{2 \cdot \# S_{l}} \\
& \leq 2^{\# A}+W \cdot \sum_{l=1}^{N} 2^{2 \cdot \# S_{l}} \\
& \leq 2 \cdot|A c t|+W \cdot \sum_{l=1}^{N} 4 \cdot\left|S_{l}\right|^{2} \\
& =O\left(|A c t|+W \cdot \sum_{l=1}^{N}\left|S_{l}\right|^{2}\right)
\end{aligned}
$$

The rest of the proof is given in the appendix. It calculates $W$ to be $4 \cdot 2^{|A c t|}$ which in turn proves the stated bound.

For a fixed set of actions and $n:=\max _{i}\left|S_{i}\right|$ this bound is simply $O\left(N \cdot n^{2}\right)$ which compares favourably to the straightforward worst case bound $O\left(n^{N+2} \cdot N^{2}\right)$ for the number of transitions in $T_{1}|\ldots| T_{N}$.

If we know more on the structure of the transition systems we are able to give the following tighter bound. See the appendix for details. 
Corollary 6.2 For $T_{i}$ and $B$ as above let $c$ be the number of sets of visible actions which occur in transitions between any two states of any component, i.e.

$$
c:=\left|\left\{A \subseteq A c t \mid \exists j \in\{1, \ldots, N\}: \exists r, s \in S_{j}: A=\left\{\alpha \mid \alpha \neq \tau \wedge\langle r, \alpha, s\rangle \in D_{j}\right\}\right\}\right| .
$$

If no component contains a visible self-loop, i.e. if for all $j \in\{1, \ldots, N\}$ there exists no $s \in S_{j}$, $\alpha \in A c t-\{r\}$ such that $\langle s, \alpha, s\rangle \in D_{j}$, then

$$
|B|=O\left((c+|A c t|) \cdot \sum_{i=1}^{N}\left|S_{i}\right|^{2}\right) .
$$

We expect $c$ to be close to $|A c t|$ in typical cases.

Let us remark that the same bounds are true for any relabelling of the $B_{i}$ 's and for arbitrary restrictions (as for example in $\left.\left(\left(B_{1} \mid B_{2}\right) \backslash A_{1} \mid\left(B_{3} \mid B_{4}\right) \backslash A_{2}\right) \backslash A_{3}\right)$. In particular this comprises the most important practical case given at the beginning of the previous section.

\section{Implementation}

We have implemented the described generation of BDDs for elementary transition systems and the operators of CCS parallel composition, restriction, and relabelling. Furthermore we implemented the symbolic model checker of Burch et al. [2]. The implementations are based on a Prolog system extended by unification in finite algebras [8].

Our implementation captures the powerful version of the $\mu$-calculus as used in [2]. In particular the $\mu$-calculus formulas for strong and weak bisimilarity can be checked. Note that special care is needed for the ordering of the boolean variables encoding the variables in these $\mu$-calculus formulas in order to avoid exponential explosion of the involved BDDs.

\section{Example}

Milner's example of a simple distributed scheduler has become a benchmark for process algebra tools $[7,9]$. The scheduler consists of one starter process and $N$ processes which are scheduled. The communication is organized in a ring. Expressed in CCS [10] the processes are as follows.

$$
\begin{array}{rll}
\text { Starter } & \stackrel{\text { def }}{=} \overline{c_{0}} \cdot 0 \\
C_{0} & \stackrel{\text { def }}{=} & c_{0} \cdot a_{0} \cdot\left(\tau \cdot \overline{c_{1}} \cdot C_{0}+\overline{c_{1}} \cdot \tau \cdot C_{0}\right) \\
C_{1} & \stackrel{\text { def }}{=} & c_{1} \cdot a_{1} \cdot\left(\tau \cdot \overline{c_{2}} \cdot C_{1}+\overline{c_{2}} \cdot \tau \cdot C_{1}\right) \\
\vdots & & \\
C_{N-1} & \stackrel{\text { def }}{=} & c_{N-1} \cdot a_{N-1} \cdot\left(\tau \cdot \overline{c_{0}} \cdot C_{N-1}+\overline{c_{0}} \cdot \tau \cdot C_{N-1}\right)
\end{array}
$$

Each cycler process $C_{i}$ awaits the permit $c_{i}$ to start, performs action $a_{i}$, and passes the permit to the next cycler either before or after some internal computation. The transition system for $C_{0}$ is given in Fig. 3. The compound process for $N$ cyclers is

$$
S C H E D_{N} \stackrel{\text { def }}{=}\left(\text { Starter }\left|C_{0}\right| C_{1}|\ldots| C_{N-1}\right) \backslash\left\{c_{0}, \ldots, c_{N-1}\right\}
$$

it is weakly bisimilar to $S P E C_{N} \stackrel{\text { def }}{=} a_{0} . a_{1} \ldots a_{N-1} . S P E C_{N}$.

Table 1 shows the size of the BDD for $S C H E D_{N}$ and the times needed to check the weak bisimilarity between $S C H E D_{N}$ and $S P E C_{N}$ compared to conventional tools. The number of states and transitions for $N>12$ as well as the times for the conventional tools for $N=20$ are extrapolated estimates. The times for AUTO and BB are taken from [9], those for Aldébaran from [7]. Times for our system were obtained on an Apollo with $16 \mathrm{MBs}$ memory. It is approximately as fast as a SUN $3 / 60$.

To be better comparable one would have to add the times needed for computing the transition relation of $S C H E D_{N}$ to the columns for AUTO, Aldébaran, and BB. Additionally the time needed to compute the transitive closure with respect to $\tau$-transitions has to be added for AUTO and Aldébaran, it is not needed for branching bisimulation. Only the column for this paper includes all these times.

Applying Corollary 6.2 to $S C H E D_{N}$ yields $c=O(|A c t|)$ and hence the size of the BDD representing the transition system of $S C H E D_{N}$ is $O\left(N \cdot \sum_{i=0}^{N-1}\left|S_{i}\right|^{2}\right)=O\left(N^{2}\right)$. 


\begin{tabular}{|r|r|r|r||r|r|r|l|}
\hline $\mathrm{N}$ & states & transitions & $\begin{array}{r}\text { nodes } \\
\text { in } \\
\text { BDD }\end{array}$ & $\begin{array}{r}\text { AUTO } \\
{[4]}\end{array}$ & $\begin{array}{r}\text { Aldébaran } \\
{[7]}\end{array}$ & $\begin{array}{r}\text { BB } \\
{[9]}\end{array}$ & \\
& & & & & & \\
\hline 6 & 577 & 2017 & 424 & $3.3 \mathrm{~s}$ & $1.9 \mathrm{~s}$ & $0.2 \mathrm{~s}$ & $177 \mathrm{~s}$ \\
8 & 3073 & 13825 & 654 & $57 \mathrm{~s}$ & $24 \mathrm{~s}$ & $1.2 \mathrm{~s}$ & $345 \mathrm{~s}$ \\
10 & 15361 & 84481 & 908 & - & - & $7.4 \mathrm{~s}$ & $665 \mathrm{~s}$ \\
12 & 73729 & 479233 & 1213 & - & - & $53 \mathrm{~s}$ & $1147 \mathrm{~s}$ \\
14 & 300000 & 2000000 & 1549 & - & - & - & $1928 \mathrm{~s}$ \\
16 & 1200000 & 8000000 & 1960 & - & - & - & $2972 \mathrm{~s}$ \\
18 & 4800000 & 32000000 & 2337 & - & - & - & $3259 \mathrm{~s}<1 \mathrm{~h}$ \\
20 & 18000000 & 128000000 & 2810 & $\left(10^{9} \mathrm{~s}\right)$ & $\left(7 \cdot 10^{7} \mathrm{~s}\right)$ & $(50000 \mathrm{~s})$ & $4927 \mathrm{~s}<1.5 \mathrm{~h}$ \\
\hline
\end{tabular}

Table 1: Benchmarks for the scheduler

\section{Conclusion}

We have presented the missing link, namely the generation of transition relations as BDDs, for exploiting symbolic model checking as a tool for process algebras. The example shows that processes out of the reach of conventional tools can be checked for bisimilarity this way.

We have shown that the BDD for $N$ parallel transition systems grows only linearly in $N$. Such a worst case bound for BDDs is rare in the literature. On the other hand it is not clear how the BDDs grow during the symbolic model checking.

Another open problem is the question whether other process algebra operators, in particular recursion can be performed on BDDs similarly efficiently.

Acknowledgement We thank Peter Warkentin, his fast implementation of operations on BDDs is used for the larger experiments reported in Table 1.

\section{Appendix}

This appendix shows how to calculate the bound $W$ needed for the proof of Theorem 6.1. For the

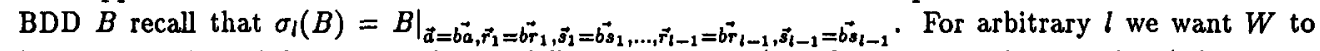
be an upper bound for the number of different relations (i.e. characteristic functions) $\sigma_{l}(B)$ for any instantiation $\sigma_{l}=\left\langle\overrightarrow{b a}, \overrightarrow{b r}_{1}, \overrightarrow{b s}_{1}, \ldots, \overrightarrow{b r}_{l-1}, \overrightarrow{b s}_{l-1}\right\rangle$.

In the following the relations $\sigma_{l}(B)$ will be stated in terms of the relations $s t a b_{l}, \operatorname{movel}_{l}(A)$ and SyncAsyncl. The relation $s t a b_{l}$ expresses that all components $T_{i}$ on level $l$ or lower are stable, i.e. perform no change of state.

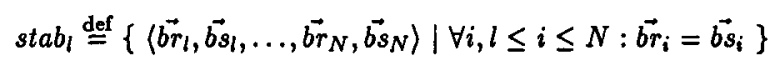

For a set $A$ of actions move $(A)$ expresses that one component $T_{j}$ on level $l$ or lower performs a transition with an action of $A$ while all other processes are stable.

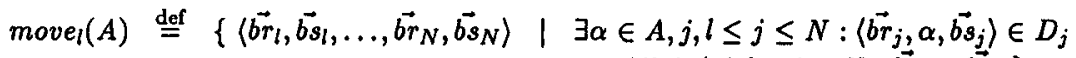

$$
\begin{aligned}
& \left.\wedge \forall i, i \neq j, l \leq i \leq N: \overrightarrow{b r_{i}}=\overrightarrow{b s_{i}}\right\}
\end{aligned}
$$

The relation SyncAsync $c_{l}$ states that some process $T_{j}$ on level $l$ or lower performs an asynchronous $\tau$-transition or that two processes $T_{j}, T_{k}$ perform synchronizing transitions. In both cases all other processes are stable.

$$
\begin{aligned}
& \text { SyncAsync } \stackrel{\text { def }}{=} \operatorname{movel}(\{\tau\}) \cup\left\{\left\langle\overrightarrow{b r}_{l}, \overrightarrow{b s_{l}}, \ldots, \overrightarrow{b r}_{N}, \overrightarrow{b s}_{N}\right\rangle \mid \exists \alpha \in A c t, j, k, j \neq k, l \leq j, k \leq N:\right. \\
& \left\langle\overrightarrow{b r}_{j}, \alpha, \overrightarrow{b s}_{j}\right\rangle \in D_{j} \wedge\left\langle\overrightarrow{b r}_{k}, \vec{\alpha}, \overrightarrow{b s}_{k}\right\rangle \in D_{k} \wedge \\
& \left.\forall i, k \neq i \neq j, l \leq i \leq N: \overrightarrow{b r}_{i}=\overrightarrow{b s_{i}}\right\}
\end{aligned}
$$


Instantiations $\sigma_{l}$ can be divided into non- $\tau$ - and $\tau$-transitions (cases $\mathbf{a}$ and $\mathbf{b}$ below). The former are always asynchronous. Depending on the number of unstable components in $\sigma_{l}$ (a component $k$ of $\sigma_{l}$ is unstable if $\overrightarrow{b r_{k}} \neq \overrightarrow{b s_{k}}$ ) we distinguish the cases a0 (no unstable components), a1 (one unstable component), and a2 (two or more unstable components).

Case a $\overrightarrow{b a} \neq \tau$

Case a0 $\overrightarrow{b r_{i}}=\overrightarrow{b s_{i}}$ for all $i \in\{1, \ldots, l-1\}$

$$
\sigma_{l}(B)= \begin{cases}\operatorname{move}_{l}(\{\overrightarrow{b a}\}) \cup \text { stabl }_{l} & \text { if } \exists j, 1 \leq j<l:\left\langle\overrightarrow{b r}_{j}, \overrightarrow{b a}, \overrightarrow{b r_{j}}\right\rangle \in D_{j} \\ \operatorname{movel}_{l}(\{\overrightarrow{b a}\}) & \text { otherwise }\end{cases}
$$

Case al $\exists j: b \vec{r}_{j} \neq \overrightarrow{b s}_{j}$ and $\forall i, i \neq j: \overrightarrow{b r}_{i}=\overrightarrow{b s}_{i}$ where $1 \leq i, j<l$

$$
\sigma_{l}(B)= \begin{cases}s t a b_{l} & \text { if }\left\langle\overrightarrow{b r}_{j}, \overrightarrow{b a}, \overrightarrow{b s}_{j}\right\rangle \in D_{j} \\ \emptyset & \text { otherwise }\end{cases}
$$

Case a2 $\exists j, k, j \neq k: \overrightarrow{b r}_{j} \neq \overrightarrow{b s}_{j} \wedge \overrightarrow{b r}_{k} \neq \overrightarrow{b s}_{k}$ where $1 \leq j, k<l$

$$
\sigma_{l}(B)=\emptyset
$$

The $\tau$-transitions are more complicated because we have to consider transitions resulting from two synchronizing processes as well as asynchronous $r$-transitions of a single process. Depending on the number of unstable components in $\sigma_{l}$ we distinguish cases b0, b1, b2 and b3. The case b3 covers those $\sigma_{l}$ which contain three or more unstable components.

The cases b0, b1 and b2 have to check whether for $\sigma_{l}$ a process can proceed asynchronously and whether two processes can synchronize. For this purpose we define the predicates Async- $\tau$ and Sync- $\tau$.

$$
\begin{aligned}
& \text { Async- } \tau(j) \quad \text { def }\left\langle\overrightarrow{b r}_{j}, \tau, \overrightarrow{b s}\right\rangle \in D_{j} \\
& \text { Sync- } \tau(j, k) \text { def } \exists \alpha \in A c t:\left\langle\overrightarrow{b r}_{j}, \alpha, \overrightarrow{b s}_{j}\right\rangle \in D_{j} \wedge\left\langle\left\langle\overrightarrow{b r}_{k}, \bar{\alpha}, \overrightarrow{b s}_{k}\right\rangle \in D_{k}\right.
\end{aligned}
$$

Case $b \overrightarrow{b a}=\tau$

\section{Case b3 $\exists M \subseteq\{1, \ldots, l-1\},|M|>2: \forall i \in M: \overrightarrow{b r}_{i} \neq \overrightarrow{b s}_{i}$}

This means that three or more processes perform a transition in $B$, which is impossible from the definition of the parallel composition operator.

$\sigma_{l}(B)=\emptyset$

Case b2 $\exists j, k, j \neq k: \vec{b}_{j} \neq \overrightarrow{b s}_{j}, \overrightarrow{b r}_{k} \neq \overrightarrow{b s}_{k}, \forall i, k \neq i \neq j: \overrightarrow{b r}_{i}=\overrightarrow{b s}_{i}$ where $1 \leq i, j, k<l$

If two processes are making a transition they are synchronizing, i.e. the remaining components must be stable.

$\sigma_{l}(B)= \begin{cases}s t a b_{l} & \text { if Sync- } \tau(j, k) \\ \emptyset & \text { otherwise }\end{cases}$

Case b1 $\exists j: \overrightarrow{b r}_{j} \neq \overrightarrow{b s}_{j} \forall i, i \neq j: \overrightarrow{b r}_{i}=\overrightarrow{b s}_{i}$ where $1 \leq i, j<l$

If process $T_{j}$ can perform an asynchronous $r$-transition or synchronize with a process $T_{i}, i<l$ the relation $s t a b_{l}$ is contained in $\sigma_{l}(B)$. The other part of $\sigma_{l}(B)$ stems from synchronisations of $T_{j}$ with a process $T_{i}, i \geq l$.

For the latter case we define the set $A_{1}:=\left\{\bar{\alpha} \mid\left\langle\overrightarrow{b r}_{j}, \alpha, \overrightarrow{b s}_{j}\right\rangle \in D_{j} \wedge \alpha \neq \tau\right\}$.

$\sigma_{l}(B)= \begin{cases}\operatorname{move}_{l}\left(A_{1}\right) \cup s t a b_{l} & \text { if Async- } \tau(j) \text { or } \exists k, j \neq k<l: \text { Sync- } \tau(j, k) \\ \operatorname{move}_{l}\left(A_{1}\right) & \text { otherwise }\end{cases}$

Case bo $\overrightarrow{b r}_{i}=\overrightarrow{b s}_{i}$ for all $i \in\{1, \ldots, l-1\}$

Again we have the same possibilities as in case b1, but the value of $j$ is not fixed, it may be in the range of 1 to $l-1$. As components 1 to $l-1$ are stable we also have the possibility that processes $T_{l}, \ldots, T_{N}$ perform an asynchronous transition or a pair of synchronizing transitions.

Let $A_{0}:=\left\{\bar{\alpha} \mid \exists j, 1 \leq j<l:\left\langle\overrightarrow{b r}_{j}, \alpha, \overrightarrow{b r_{j}}\right\rangle \in D_{j} \wedge \alpha \neq \tau\right\}$. $\sigma_{l}(B)=\left\{\begin{array}{cc}\text { move }_{l}\left(A_{0}\right) \cup S_{\text {SyncAsync }} & \text { if } \exists j, 1 \leq j<l: \text { Async- } \tau(j) \text { or } \\ \cup \text { stab }_{l} & \exists j, k, j \neq k, 1 \leq j, k<l: \text { Sync- } \tau(j, k) \\ \text { move }_{l}\left(A_{0}\right) \cup \text { SyncAsync } & \text { otherwise }\end{array}\right.$ 
By counting all possibilities for $\sigma_{l}(B)$ in cases a0 to a2 and bo to b3 and allowing any subset of Act for $A_{0}$ and $A_{1}$ we get the upper bound $W=4 \cdot 2^{|A c t|}$.

Critical cases for a better approximation are b0 and b1 from which we can get a smaller upper bound $W$. If no process contains a visible self-loop the set $A_{0}$ in case bo is empty. We then get $W=2 \cdot|A c t|+4+2 \cdot c$ with $c$ as stated in Corollary 6.2 .

\section{References}

[1] R. E. Bryant. Graph-based algorithms for boolean function manipulation. IEEE Transactions on Computers, C-35(8):677-691, 1986.

[2] J. R. Burch, E. M. Clarke, K. L. McMillan, D. L. Dill, and L. J. Hwang. Symbolic model checking: $10^{20}$ states and beyond. In Proceedings of the 5th IEEE Symposium on Logic in Computer Science, Philadelphia, pages 428-439, 1990.

[3] R. Cleaveland, J. Parrow, and B. Steffen. The concurrency workbench. In J. Sifakis, editor, Automatic Verification Methods for Finite State Systems. Proceedings, Grenoble, 1989, volume 407 of Lecture Notes in Computer Science, pages 24-37, Berlin et al., 1990. Springer.

[4] R. de Simone and D. Vergamini. Abord auto. Rapports Techniques 111, INRIA, Sophia Antipolis, 1989.

[5] E. A. Emerson and C.-L. Lei. Efficient model checking in fragments of the propositional mucalculus. In Proc. of the First Annual Symp. on Logic in Computer Science, pages 267-278. Computer Society Press, 1986.

[6] K. Estenfeld, H.-A. Schneider, D. Taubner, and E. Tidén. Computer aided verification of parallel processes. In A. Pfitzmann and E. Raubold, editors, VIS ' 91 Verläßliche Informationssysteme. Proceedings, Darmstadt 1991, volume 271 of Informatik Fachberichte, pages 208-226, Berlin, 1991. Springer.

[7] J.-C. Fernandez. An implementation of an efficient algorithm for bisimulation equivalence. Science of Computer Programming, 13:219-236, 1989/90.

[8] T. Filkorn. Unifikation in endlichen Algebren und ihre Integration in Prolog, Master's Thesis, Techn. Universität München, 1988.

[9] J. F. Groote and F. Vaandrager. An efficient algorithm for branching bisimulation and stuttering equivalence. In ICALP '90, Lecture Notes in Computer Science, Berlin, 1990. Springer.

[10] R. Milner. Communication and Concurrency. Prentice Hall, New York, 1989.

[11] C. Stirling and D. Walker. Local model checking in the modal mu-calculus. In J. Díaz and F. Orejas, editors, TAPSOFT '89. Volume 1., Proceedings, Barcelona 1989, volume 351 of Lecture Notes in Computer Science, pages 369-383, Berlin, 1989. Springer.

[12] D. Taubner. Finite Representations of CCS and TCSP Programs by Automata and Petri Nets, volume 369 of Lecture Notes in Computer Science. Springer, Berlin, 1989. 